\title{
"ESTUDIO DE LA RESISTENCIA A ANTIMICROBIANOS EN CEPAS DE Enterococcus spp, AISLADAS DE AVES Y CERDOS DE PRODUCCIÓN".
}

\author{
Autores: Lapierre, Lisette ${ }^{1^{*}}$, Toro Cecilia ${ }^{2}$., San Martín, Betty ${ }^{3}$.
}

1*: Departamento de Salud Ambiental, Instituto de Salud Pública de Chile. email: llapierre@ispch.cl. Marathon 1000, Ñuñoa, Santiago. 56 (2) 5755545

2: Laboratorio de Antibióticos, Programa de Microbiología y Micología del ICBM, Facultad de Medicina Universidad de Chile.

3: Laboratorio de Farmacología, Facultad de Ciencias Veterinarias y Pecuarias, Universidad de Chile. Santa Rosa 11735, La Pintana, Santiago.

\section{Resumen}

La resistencia en las cepas bacterianas aparece junto con el uso de los antimicrobianos. Hoy en día el uso masivo de estos fármacos en el hombre, los animales y la agricultura ha transformado este fenómeno en un problema creciente, que involucra cada día mayor número de cepas, nuevas especies bacterianas y nuevos mecanismos. En este sentido, las bacterias son particularmente eficientes en aumentar los efectos de la resistencia, no sólo por su habilidad de multiplicarse rápidamente sino también, por su capacidad de transferir genes en forma horizontal. En este sentido la importancia del género Enterococcus radica en su resistencia natural a múltiples antibióticos y a su capacidad de adquirir nueva resistencia incluida a la vancomicina.

El objetivo de este estudio fue caracterizar fenotípica y genotípicamente la resistencia a diversos antimicrobianos en cepas indicadoras Enterococcus spp aisladas desde animales sanos de importancia económica, como son las aves y los cerdos. Se determinaron los porcentajes de resistencia frente a distintos antimicrobianos mediante el método de dilución en placa o concentración mínima inhibitoria (CIM) y posteriormente se definió el mecanismo genético asociado a la resistencia a eritromicina.

Los resultados de este estudio entregan información de la situación de la resistencia en cepas de Enterococcus spp aisladas de dos especies animales productoras de alimento 
de alto consumo en nuestro país. Por lo que estos resultados podrían servir para que los médicos veterinarios realicen una mejor utilización de los antimicrobianos en las distintas producciones lo que podría tener un impacto en salud pública.

\begin{abstract}
The appearance of resistance bacterial strains appears concurrent with the use of antimicrobial drugs. Within recent times the progressively increasing use of the drugs in humans, animals and the agriculture have transformed this phenomena into a growing problem, that every day involves new number of strains, new bacterial species and mechanisms. In this context, bacteria are particularly efficient in developing greater resistance, not only because of their ability to rapidly multiply but also their capacity to transfer genes in a horizontal form. In this respect the importance of Enterococcus is natural resistance to multiple antibiotics and this aptitude to acquire new resistance included to the vancomycin.
\end{abstract}

The objective of this study was to phenotypically and genotypically characterize the antimicrobial resistance in indicator strains Enterococcus spp isolated from healthy animals of economic importance, such as poultry and pigs.

Firstly the percentage of resistance to the different antimicrobial drugs was determined by the method of dilution in plate (MIC), then the genetic mechanisms associated with resistance to erythromycin was defined.

The results of this study give up to date information on the situation regarding bacterial resistance in Enterococcus strains isolated in two animal species producing of food which are highly consumed in our country. This information should be taken account in the development of public health policy for the use of antimicrobials in food producing animal with the aim of protecting human health. 


\section{Introducción:}

Enterococcus es una bacteria Gram-positiva que forma parte de la microbiota intestinal de humanos y animales (Juliet 2002). Son capaces de sobrevivir en medios poco enriquecidos como agua, suelo, alimentos y permanecen en superficies inanimadas por 24 horas. A pesar de que no es un patógeno primario, en la actualidad a emergido como un importante causa de enfermedad nosocomial (Silva et al, 2006). Son causantes en pacientes humanos de infección del tracto urinario, endocarditis infecciosa, bacteremias e infecciones intrahospitalarias asociadas a procedimientos (Juliet, 2002). En Estados Unidos Enterococcus spp., causa más del 12\% de las infecciones nosocomiales y es uno de los tres patógenos más comúnmente aislados en las infecciones intrahospitalarias (Shepard and Gildmore, 2002). El antibiótico de elección es la ampicilina y en enfermedades graves puede asociarse con aminoglucósidos siempre que la resistencia sea de bajo nivel. Finalmente como última opción se utiliza vancomicina (Juliet, 2002). La vigilancia de la resistencia en cepas de Enterococcus aisladas de animales de producción es muy importante para evitar que cepas resistentes puedan transferir sus determinantes de resistencia a otras cepas potencialmente patógenas, o que estas mismas cepas provoquen en pacientes susceptibles infecciones, es por este motivo que conocer el porcentaje de resistencia en cepas de origen veterinario permitiría tomar las medidas tendientes a disminuir el riesgo de traspaso de cepas resistentes a la población.

En medicina veterinaria los antimicrobianos son esenciales para el tratamiento de las enfermedades infecciosas, pero su uso excesivo puede conducir a la selección de resistencia. En la actualidad, en nuestro país existe escasa información acerca de cuáles son los antimicrobianos que muestran mayores porcentajes de resistencia y de los mecanismos genéticos involucrados en conferir esta característica en las cepas bacterianas de Enterococcus aislados desde animales de producción.

El fenómeno de resistencia bacteriana no es nuevo, y se evidenció poco tiempo después de la utilización clínica de la penicilina en pacientes humanos. En 1990 emergen cepas de Enterococcus spp resistentes a ampicilina y diversas bacterias multi-resistentes que dificultan los tratamiento con impacto en la mortalidad, lo que pone en evidencia la gravedad del problema (Power, J.; 2004; Martel et al, 2000). 
Estudios relacionados con la emergencia, transferencia y diseminación de microorganismos resistentes a antimicrobianos, han señalado que existen ciertos factores que favorecen el desarrollo y diseminación de la resistencia bacteriana (Levy and Marshall, 2004; Phillips et al, 2004). Entre los factores se ha señalado el tipo de antimicrobiano y el modo de utilización de éste en los diferentes planteles de producción animal. Estudios realizados en diversos países de la Unión Europea, demostraron que cepas de Enterococcus faecium aisladas de muestras de distintos animales de producción como cerdos, aves y bovinos presentaban niveles de resistencia a vancomicina, antimicrobiano de uso exclusivo en medicina humana (Aarestup et al, 2000; Sander, H.; 2000). Esta situación, determinó que la Comunidad Europea a partir del año 1997 revocara la autorización de la utilización de avoparcina, un promotor de crecimiento del mismo grupo de la vancomicina, como medida con carácter cautelar y que en el año 1999 se eliminaran los registros para los antimicrobianos bacitracina, espiramicina, virginiamicina, y fosfato de tilosina como promotores del crecimiento, debido al potencial riesgo en salud pública (Casewell et al, 2003; Schwarz and ChaslusDancla, 2001). Por otra parte, la utilización de antimicrobianos contribuye a la contaminación del medio ambiente con microorganismos resistentes (Phillips et al, 2004; Campagnolo et al, 2002). Es así como, los residuos de antimicrobianos utilizados en animales pueden permanecer en sus productos y/o pueden ser vertidos a nuestro medio contaminándolo.

En nuestro país se han realizado estudios para determinar la resistencia fenotípica y genotípica en cepas aisladas desde animales. Las cepas más frecuentemente estudiadas han sido E. coli o Salmonella spp, encontrándose altos porcentajes de resistencia a los antimicrobianos más comúnmente utilizados (Lapierre et al, 2008; Lapierre et al, 2010). Sin embargo, existe escasa información sobre la prevalencia de la resistencia de cepas de Enterococcus spp aisladas desde animales de producción por lo que el propósito de este trabajo fue caracterizar la resistencia a antimicrobianos en cepas indicadoras de Enterococcus spp aisladas desde animales sanos de importancia económica, como son las aves y los cerdos. 


\section{Material y Métodos:}

Grupo de animales: Se trabajó con cerdos y aves faenados en mataderos de la región metropolitana y sus alrededores, desde las cuales se tomaron muestras de heces para realizar el aislamiento bacteriano.

Recolección de las muestras: Las muestras fueron recolectadas entre marzo del 2003 y diciembre del 2004. Los animales fueron muestreados al azar, con un total de 10 muestras máximo por plantel. Para los cerdos las muestras $(n=192)$ fueron tomadas desde el intestino grueso en la zona de evisceración de la planta faenadora. Se tomaron $5 \pm 0,5$ gramos de contenido fecal depositándose en frascos estériles, los cuales contenían medio de transporte Cary-Blair. Para el caso de las aves $(\mathrm{n}=152)$, las muestras fueron tomadas con una tórula estéril a nivel cloacal desde corrales de diferentes planteles avícolas. Luego la muestra fue depositada en un tubo estéril que contenía medio de transporte Cary-Blair. Las muestras fueron transportadas al laboratorio refrigeradas para ser analizadas dentro de 24 horas posterior a su recolección.

Aislamiento y caracterización de las cepas de Enterococcus spp: Las muestras recolectadas se sembraron directamente en agar M-Enterococcus incubándose en estufa de cultivo a $37{ }^{\circ} \mathrm{C} \pm 1{ }^{\circ} \mathrm{C}$ por 24 a 48 horas. Pasado este tiempo, a las colonias sospechosas de color blanco, se les realizó las pruebas bioquímicas estándares para su identificación de género: hidrólisis de agar bilis-esculina y crecimiento en $\mathrm{NaCl}$ al $6,5 \%$. Una vez identificadas las cepas se guardaron en tubos que contienen medio Tripticasa Soya Base (TSB) y $15 \%$ de glicerol y fueron congeladas a $-70^{\circ} \mathrm{C}$.

Pruebas de susceptibilidad in Vitro: Se utilizó la técnica de la concentración mínima inhibitoria (CIM), siguiendo las normas recomendadas por el Clinical and Laboratory Standards Institute CLSI, 2006 y por el DANMAP, 2002.Como cepa control se utilizó E. faecalis ATCC 29212. Se analizaron los siguientes antimicrobianos: Penicilina $\mathrm{G}^{(\text {(Arlab) }}$, Vancomicina ${ }^{\text {(Sigma) }}$, Gentamicina de alta carga $^{\text {(Arlab), Eritromicina }}{ }^{\text {(Arlab) }}$, Oxitetraciclina $^{(\text {Arlab) }}$, Cloranfenicol. ${ }^{\text {(Sigma) }}$

Determinación de genes de resistencia a eritromicina mediante PCR: Para determinar la presencia de los genes de resistencia, presentes en las cepas aisladas se utilizó la 
técnica de reacción de la polimerasa en cadena o PCR. Los partidores y las condiciones para la amplificación utilizadas en este estudio se encuentran descritos en la Tabla 1. Como control negativo se utilizaron cepas sensibles según los valores de CIM, aisladas en este estudio y como controles positivos se utilizaron cepas de Streptococcus spp, que presentaban los genes buscados.

$\underline{\text { Tabla 1: }}$ Condiciones de amplificación utilizadas para detectar genes de resistencia a eritromicina.

\begin{tabular}{ccccc}
\hline Antimicrobiano & Gen & $\begin{array}{c}\text { Tamaño } \\
\text { Producto } \\
(\mathrm{pb})\end{array}$ & $\begin{array}{c}\text { Temp. } \\
\text { Hibridación }\end{array}$ & $\begin{array}{c}\text { Secuencia de Partidores }\left(^{*}\right) \\
\left(5^{\prime} \text { a } 3^{\prime}\right)\end{array}$ \\
\hline Eritromicina & ermB & 616 & $50^{\circ} \mathrm{C}$ & $\begin{array}{c}\text { CGAGTGAAAAAGTACTCAACC } \\
\text { GGCGTGTTTCATTGCTTGATG }\end{array}$ \\
& & & & \\
Eritromicina & mefA & 348 & $50^{\circ} \mathrm{C}$ & AGTATCATTAATCACTAGTGC \\
& & & TTCTTCTGGTACTAAAAGTGG \\
Eritromicina & ermA & 206 & $50^{\circ} \mathrm{C}$ & GCATGACATAAACCTTCA \\
& & & AGGTTATAATGAAACAGA
\end{tabular}

\section{Resultados:}

Determinación de la resistencia fenotípica a los antimicrobianos:

Se aislaron un total de 96 cepas en aves y 81 cepas en cerdos, a las cuales se les realizaron las pruebas de concentración mínima inhibitoria o CIM. Los resultados fueron interpretados según las normas establecidas por el Clinical and Laboratory Standards Institute (CLSI) y DANMAP para definir las cepas resistentes a cada antimicrobiano. Se estimaron los porcentajes de susceptibilidad in vitro en cepas Enterococcus spp. Tetraciclina y eritromicina presentaron en ambas especies animales valores de $\mathrm{CIM}_{90}$ mayores a $128 \mu \mathrm{g} / \mathrm{ml}$ por lo que estos antimicrobianos carecen de eficacia in vitro en estas cepas, así también se encontraron niveles de $\mathrm{CIM}_{90}$ altos para penicilina especialmente en las cepas aisladas de aves. Ninguna cepa fue resistente a vancomicina, gentamicina, cloranfenicol (Tabla 2). 
Tabla 2: CIM $_{50}$ y CIM $_{90}$ obtenidos en cepas de Enterococcus spp aisladas de aves y cerdos.

\begin{tabular}{|c|c|c|c|c|c|c|c|}
\hline \multicolumn{2}{|c|}{$\begin{array}{c}\text { Antimicrobianos } \\
\text { Punto de Corte }(\mu \mathrm{g} / \mathrm{ml})\end{array}$} & \multicolumn{3}{|c|}{ AVES n=96 } & \multicolumn{3}{c|}{ CERDOS n=81 } \\
\cline { 3 - 8 } \multicolumn{2}{|c|}{} & $\begin{array}{c}\mathrm{CIM}_{50} \\
(\mu \mathrm{g} / \mathrm{ml})\end{array}$ & $\begin{array}{c}\mathrm{CIM}_{90} \\
(\mu \mathrm{g} / \mathrm{ml})\end{array}$ & $\begin{array}{c}\% \text { Cepas } \\
\text { Resistentes }\end{array}$ & $\begin{array}{c}\mathrm{CIM}_{50} \\
(\mu \mathrm{g} / \mathrm{ml})\end{array}$ & $\begin{array}{c}\mathrm{CIM}_{90} \\
(\mu \mathrm{g} / \mathrm{ml})\end{array}$ & $\begin{array}{c}\% \text { Cepas } \\
\text { Resistentes }\end{array}$ \\
\hline Tetraciclina & $\geq 16$ & 64 & $\geq 128$ & $74 \%$ & $\geq 128$ & $\geq 128$ & $82 \%$ \\
\hline Penicilina & $\geq 16$ & $\leq 0,125$ & 16 & $22 \%$ & $\leq 0,125$ & 4 & $19 \%$ \\
\hline Vancomicina & $\geq 32$ & $\leq 0,125$ & $\leq 0,125$ & $0 \%$ & $\leq 0,125$ & $\leq 0,125$ & $0 \%$ \\
\hline Eritromicina & $\geq 8$ & 16 & $\geq 128$ & $37 \%$ & 64 & $\geq 128$ & $56 \%$ \\
\hline Gentamicina & $\geq 500$ & $\leq 500$ & $\leq 500$ & $0 \%$ & $\leq 500$ & $\leq 500$ & $0 \%$ \\
\hline Cloranfenicol & $\geq 32$ & 2 & 4 & $0 \%$ & 4 & 8 & $0 \%$ \\
\hline $\begin{array}{c}\% \\
\text { Resistencia }\end{array}$ & ---- & ----- & ---- & $85 \%$ & ----- & ----- & $94 \%$ \\
\hline
\end{tabular}

La multi-resistencia, en los aislados de aves el porcentaje alcanzó el 44,7\% y en los aislados de cerdos el 56,7\%, siendo el perfil de multi-resistencia más frecuente en ambas poblaciones tetraciclina/eritromicina (Tabla 3 ).

Tabla 3: Perfiles de multi-resistencia en las cepas de Enterococcus spp aisladas de aves y de cerdos.

\begin{tabular}{|c|c|c|}
\hline $\begin{array}{c}\text { Perfiles de Multi-resistencia en cepas de } \\
\text { Enterococcus spp }\end{array}$ & $\begin{array}{c}\mathrm{N}^{\circ} \text { Cepas } \\
\text { Multi-resistentes Aves }\end{array}$ & $\begin{array}{c}\mathrm{N}^{\circ} \text { Cepas } \\
\text { Multi-resistentes Cerdos }\end{array}$ \\
\hline Tetraciclina+ Eritromicina & 35 & 45 \\
\hline Tetraciclina + Penicilina & 2 & 0 \\
\hline Tetraciclina + Eritromicina + Penicilina & 7 & 3 \\
\hline Total de Cepas Multi-resistentes & $43 / 93\left(44,7 \%^{*}\right)$ & $46 / 81\left(56,70^{*}\right)$ \\
\hline
\end{tabular}

Detección de Determinantes de Resistencia a Eritromicina:

Se investigó la presencia de los genes involucrados con mayor frecuencia en la resistencia a macrólidos. Estos genes son mefA, ermA y ermB. El gen mefA codifica una bomba de eflujo y los genes ermA y ermB codifican para enzimas metilasas que modifican el sitio blanco de unión del antimicrobiano. En el caso de las cepas aisladas de aves, 35 fueron resistentes a eritromicina de ellas 28 amplificaron un fragmento de $616 \mathrm{pb}$ concordante con el control positivo para el gen ermB, una cepa amplificó un fragmento de 206 pb que concuerda con ermA y otra cepa amplificó un fragmento de $348 \mathrm{pb}$ que concuerda con mefA. Con respecto a las 45 cepas resistentes a eritromicina aisladas de cerdos, 39 amplificaron para ermB, ninguna amplificó los otros dos genes. 


\section{Discusión:}

Con el objetivo de aportar información respecto a la problemática de la resistencia bacteriana en nuestro país, nosotros estudiamos la resistencia fenotípica y genotípica frente a diferentes antimicrobianos, en bacterias de Enterococcus spp aisladas desde aves y cerdos. Es importante señalar algunas consideraciones para el género Enterococcus spp, ya que este género bacteriano ha emergido en las últimas décadas como un patógeno importante asociado a infecciones nosocomiales en pacientes humanos y la resistencia a antimicrobianos en estas cepas agrava este problema (Silva et al, 2006; Arias et al, 2003; Sander, H.; 2000). Estas bacterias, presentan resistencia intrínseca a cefalosporinas, clindamicina, sulfametoxazol +trimetoprim, limitando las alternativas terapéuticas (Causse et al, 2006; Moubareck et al, 2003); por este motivo, es trascendental disminuir la prevalencia de Enterococcus spp resistentes a los antimicrobianos, especialmente a la vancomicina (Hershberger et al, 2005; De Leener et al, 2005), pudiendo transferir esta resistencia a cepas patógenas como Staphylococcus aureus meticilino resistentes (Arias et al, 2003; Sander, H.; 2000).

En nuestro estudio ninguna cepa fue resistente a vancomicina, posiblemente estos resultados se deban a que la avoparcina fue prohibida en Chile en el año 2000 para su uso en animales. Sin embargo, no puede descartarse la posibilidad de emergencia de esta resistencia y por lo tanto, esta situación debería ser vigilada permanentemente con el fin de detectar a tiempo cepas resistentes que ponen en riesgo la salud pública.

En relación al antimicrobiano gentamicina, la eficacia en el tratamiento se pierde, con $\mathrm{CIM} \geq 500 \mu \mathrm{g} / \mathrm{ml}$ (Aslangul et al, 2005). Los CIM frente a gentamicina en nuestras cepas fueron menores a $500 \mu \mathrm{g} / \mathrm{ml}$, este hecho no es de extrañar ya que la gentamicina no es un antimicrobiano muy utilizado en producción animal principalmente por sus efectos adversos y porque se administra en forma inyectable. Con respecto a la penicilina nosotros sí encontramos cepas resistentes con $\mathrm{CIM}_{90}$ de $16 \mu \mathrm{g} / \mathrm{ml}$ en los aislados de aves y de $4 \mu \mathrm{g} / \mathrm{ml}$ en los aislados de cerdos.

Los altos niveles de resistencia a eritromicina encontrados en cepas de Enterococcus

spp aislados de aves y cerdos concuerdan con datos internacionales; la literatura señala que estos fármacos son muy utilizados en las distintas producciones pecuarias 
especialmente en la producción porcina, siendo esto quizás la principal causa de los elevados niveles de resistencia a nivel mundial (Aaerestrup et al, 2002).

En general, los genes de resistencia para eritromicina incluyen metilasas codificadas por genes del tipo erm y bombas de eflujo como mefA, siendo el gen ermB el más frecuente en cepas de Enterococcus spp resistentes (Hummel et al, 2006; Khan et al, 2002). Este gen posee la característica de encontrarse dentro de plasmidios y/o en transposones unido a otros genes de resistencia transfiriéndose en forma conjunta (Hummel et al, 2006). Nosotros estudiamos la presencia de los genes ermB, ermA y mefA en las cepas resistentes, encontrando que el gen más frecuente fue ermB, para los aislados de ambas poblaciones animales. Este gen ha sido encontrado en diferentes cepas de Enterococcus spp, Streptococcus pneumoniae, S. pyogenes, S. aureus resistentes a macrólidos y se ha asociado a CIM altos como los observados en nuestro estudio (Aarestrup et al, 2002; Portillo et al, 2000). Nueve cepas resistentes a eritromicina no presentaron ninguno de los genes estudiados; posiblemente la base molecular de esta resistencia se deba a un gen no incluido en este estudio, como ermC, ermF o a mutaciones en los nucleótidos que constituyen el sitio de unión al fármaco como: A2058, A2059 del dominio V y A725 del dominio II del RNA ribosomal (Xiong et al, 1999).

En la literatura, se ha asociado frecuentemente a los genes de resistencia a macrólidos con otros genes de resistencia especialmente para tetraciclinas (Hummel et al, 2006). Por este motivo, al seleccionar bacterias resistentes a macrólidos se podrían seleccionar a la vez cepas multi-resistentes. Esta situación se presentó en nuestras cepas, ya que todas las cepas resistentes a eritromicina también lo fueron a tetraciclina, por esta razón deberían supervisarse exhaustivamente los tratamientos con fármacos de la familia de los macrólidos.

Finalmente los resultados entregados señalan que las cepas de Enterococcus spp., aisladas desde animales productores de alimentos, presentan resistencia a alguno de los antimicrobianos incluidos en este estudio y por lo tanto se hace necesario establecer un programa de vigilancia de la resistencia en medicina veterinaria para poder diseñar pautas que controlen la emergencia y diseminación de este riesgo con el fin de resguardar el medio ambiente, la salud animal y la salud pública. 


\section{Agradecimiento:}

Los autores agradecen el financiamiento otorgado por el Proyecto FONDECYT $\mathrm{N}^{\circ}$ 1030857

Bibliografía:

AARESTRUP, F.; AGERSO, Y.; GERNER-SMIDT, P.; MADSEN, M.; JENSEN, L. 2000. Comparison of antimicrobial resistance phenotype and resistance genes in Enterococcus faecalis and Enterococcus faecium from humans in the community, broilers, pigs in Denmark. Diagnostic Microbiology infecting Disease. 37:127-137.

AARESTRUP, F.; HASMAN, H.; JENSEN, L.; MORENO, M.; HERRERO, I.; DOMINGUEZ, L.; FINN, M.; FRANKLIN, A. 2002. Antimicrobial resistance among enterococci from pigs in three European countries. Appl Environ Microbiol. 68: 4127-4129.

ARIAS, C.; REYES, J.; ZUÑ̃IGA, L.; CORTÉS, C.; CRUZ, C.; RICO, L.; PANESSO, D. 2003. Multicentre surveillance of antimicrobial resistance in enterococci and staphylococci from colombian hospitals, 2001-2002. Journal of Antimicrobial Chemotherapy. 51: 59-68

ASLANGUL, E.; RUIMY, R.; CHAU, F.; GARRY, L.; ANDREMONT, A.; FANTI, B. 2005. Relationship between the levels of acquired resistance to gentamicin and synergism with amoxicillin in Enterococcus faecalis. Antimicrobial Agents and Chemotherapy. 49: 4144-4148.

CAMPAGNOLO, E.; JONSON, K.; KARPATI, A.; RUBIN, C.; KOLPIN, D.; MEYER, M.; ESTEBAN, J.; CURRIER, R.; SMITH, K, THU, K.; MC GEEHIN, M. 2002. Antimicrobial residues in animal waste and water resources proximal to large-scale Swine and poultry. Science Total Environment. 1: 89-95. 
CASEWELL, M.; FRIIS, C.; MARCO, E. 2003. The European ban on growthpromoting antibiotics and emerging consequences for human and animal health. $J$ Antimicrob Chemothe 52: 159-161.

CAUSSE, M.; FRANCO-ALVAREZ DE LUNA, F.; GRACÍA-MAYORGAS, AD.; RODRIGUEZ, F.; CASAL, M. 2006. Sensibilidad a los antimicrobianos de Enteroccoccus faecalis aislados en pacientes de la provincia de Córdoba España. Rev. Esp. Quimioterap 19: 140-143.

CLSI. Clinical and Laboratory Standards Institute. 2006. Performance standards for Antimicrobial Susceptibility Testing; Sixteenth Informational Supplement. M100-S16. Vol $26 \mathrm{~N}^{\mathrm{o}} 3$.

DE LEENER, E.; MARTEL, A.; DE GRAEF, E.M.; TOP, J.; BUTAYE, P.; HAESEBROUCK, F.; WILLEMS, R.; DECOSTERE, A. 2005. Molecular analysis of human, porcine, and poultry Enterococcus faecium isolates and their erm(B) genes.Appl Environ Microbiol. 71:2766-2770.

EMBORG, D.; AND HEUER, E. 2002. DANMAP Use of antimicrobial agents and occurrence of antimicrobial resistance in bacteria from food animals, foods and humans in Denmark. Staten's Serum Institute, Danish Veterinary and Food Administration, Danish Medicines Agency, Danish Veterinary Institute, Copenhagen, Denmark. 2003 ; 68.

HERSHBERGER, E.; OPREA, SF.; DONABEDIAN, SM.; PERRI, M.; BOZIGAR, P.; BARTLETT, P.; ZERVOS, MJ. 2005. Epidemiology of antimicrobial resistance in enterococci of animal origin. J Antimicrob Chemother. 55:127-130.

HUMMEL, A.; HOLZAPFEL, W.; FRANZ, CH. 2007. Characterisation and transfer of antibiotic resistance genes from enterococci isolated from food. Systematic and Applied Microbiology. 30 (1): 1-7. 
JULIET CHRYSTAL. 2002. Estudio de susceptibilidad in vitro de Enterococcus spp. Revista Chilena Infectología. 19 (Supl.2): 111-115.

KHAN, A.A.; NAWAZ, M.; KHAN, A.; STEELE, R. 2002. Detection and Characterization of erythromycin-resistant methylase gene in gram-positive bacteria isolated from poultry litter. Appl. Microbiol. Biotech. 59: 377-381.

LAPIERRE, L., SAN MARTÍN B., ARAYA-JORDAN, C., BORIE C. 2010. Comparison of integron-linked antibiotic resistance genes in strain of Salmonella spp isolated from swine in Chile in 2005 and 2008. Canadian Journal. Microbiology. $56: 515-521$.

LAPIERRE, L.; CORNEJO, J.; BORIE, C.; TORO, C.; SAN MARTIN, B. 2008. Genetic Characterization of antibiotic resistance genes linked to class 1 integrons in comensal strains of Escherichia coli isolated from poultry and swine. Microbial Drug Resistance. Vol. 14, (4): 265-272.

LEVY, S. AND MARSHALL, B. 2004. Antibacterial resistance worldwide: causes, challenges and responses. Nat Med. 10 :S122-129.

MARTEL，L.; TARDY，F.; BRISABOIS，A.; LAILLER，R.; COUDERT，M.; CHASLUS-DANCLA, E. 2000. The French antibiotic resistance monitoring programmes. International Journal of Antimicrobial Agents. 14:275-283.

MOUBARECK, C.; BOURGEOIS, N.; COURVALIN， P.; DOUCETPOPULAIRE, F. 2003. Multiple Antibiotic Resistance Gene Transfer from Animal to Human Enterococci in the Digestive Tract of Gnotobiotic Mice. Antimicrobial Agents and Chemotherapy. 47: 2993-2996

PHILliPS, I.; CASEWELL, M.; COX, T.; DE GROOT, B.; FRIIS, C.; JONES, R.; NIGHTINGALE, CH.; PRESTON, R.; WADDELL, J. 2004. Does the use of antibiotics in food animals pose a risk to human health? A critical review of published data. Journal Antimicrobial Chemotherapy. 53: 28-52. 
POWER JH. 2004. Antimicrobial drug development-The past, the present and the future. Clinical Microbiology Infection. 10: 23-31.

SANDER, H. 2000. Enterococos resistentes a vancomicina ¿Infección emergente inminente? Revista Chilena de Infectología. 19 supl. 1.

SCHWARZ, S.; AND CHASLUS - DANCLA E. 2001. Use of antimicrobial in veterinary medicine and mechanisms of resistance. Veterinary Research. 32: 201-225.

SHEPARD BD AND GILDMORE MS. 2002. Antibiotic resistant enterococci: the mechanisms and dynamics of drug introduction and resistance. Microbes Infection. 4: 215-224.

SILVA, J., ASSERELlA, L., BOLADOS, N., HERRERA, N., LEYTON J. 2006. Resistencia a antimicrobianos en cepas de Enterococcus sp aisladas en hospitales del norte de Chile. Revista Chilena de Infectología. 23 (3): 226-231.

XIONG, L.; SHAH, S.; MAUVAIS, P.; MANKIN, A. 1999. A ketolide resistance mutation in domain II of 23S rRNA reveals the proximity of hairpin 35 to the peptidyl transferase centre. Molecular Microbiology. 31: 633-639. 